\title{
FRAÇÕES DE CARBONO E NITROGÊNIO EM FUNÇÃO DA TEXTURA, DO RELEVO E DO USO DO SOLO NA MICROBACIA DO AGRESTE EM VACA BRAVA (PB) ${ }^{(1)}$
}

\author{
Sandra Regina da Silva Galvão(2), Ignacio Hernan \\ Salcedo $^{(3)}$ \& Antonio Clementino dos Santos ${ }^{(4)}$
}

\begin{abstract}
RESUMO
A produtividade em sistemas agrícolas de subsistência ou de baixos insumos depende do fornecimento de nutrientes oriundos da mineralização da matéria orgânica do solo (MOS). Portanto, a quantidade e a qualidade da MOS são duas variáveis fundamentais relacionadas com a sustentabilidade da produção agropecuária de subsistência. O objetivo do presente trabalho foi analisar as inter-relações de usos do solo, as posições no relevo e suas texturas com os teores totais de $\mathrm{C}(\mathrm{Ct})$ e $\mathrm{N}(\mathrm{Nt})$, a fração de $\mathrm{C}$ oxidável por $\mathrm{KMnO}_{4} 16,5 \mathrm{~mol} \mathrm{~L}^{-1}(\mathrm{Cox})$ e a fração de $\mathbf{N}$ aminoaçúcar ( $\mathrm{N}$ amino). Esses atributos foram quantificados em 260 amostras simples de solo $(0-20 \mathrm{~cm})$ retiradas da microbacia Vaca Brava (PB), em áreas de uso agropecuário, num sistema de amostragem com arranjo fatorial de estratos. Os teores de $\mathrm{Ct}$, $\mathrm{Nt}$ e $\mathrm{N}$ amino decresceram na seqüência: pastagem $>$ capineira > roçado. Em relação ao relevo, tanto os teores de $\mathrm{Ct}$ e $\mathrm{Nt}$ quanto suas frações apresentaram os maiores teores nas áreas de várzea, siltosas e os menores nas áreas de rampa pedimentada, mais arenosas. A redistribuição de sedimentos nas vertentes foi ocasionada por processos erosivos da camada superficial das encostas, nas quais predominam texturas finas. Em relação às classes texturais, os teores de MOS e de suas frações foram maiores $(p<0,05)$ nas amostras de textura argilo-arenosa e menores nas de textura areia franca. Em todas as situações analisadas, os teores de Cox e $\mathrm{N}$ amino tenderam a acompanhar as variações nos teores de $\mathrm{Ct}$ e Nt. Por conta desta última tendência, os índices $\mathrm{Cox} / \mathrm{Ct}$ e $\mathrm{Nt} / \mathrm{N}$ amino, que representam a qualidade da MOS, indicaram poucas mudanças significativas nesse atributo de acordo com o uso, relevo ou classe textural.
\end{abstract}

Termos de indexação: agricultura familiar, fertilidade de solo, pastagens, frações lábeis de $\mathbf{C}$ e $\mathbf{N}$.

\footnotetext{
${ }^{(1)}$ Parte da Tese de Mestrado da primeira autora, apresentada ao programa de Pós-graduação em Tecnologias Energéticas e Nucleares, Universidade Federal de Pernambuco - UFPE. Recebido para publicação em agosto de 2004 e aprovado em setembro de 2005.

${ }^{(2)}$ Doutoranda do Departamento de Energia Nuclear da Universidade Federal de Pernambuco - UFPE. Av. Luiz Freire 1000, Cidade Universitária, CEP 50740-540 Recife (PE). E-mail: reginassg@uol.com.br

(3) Professor Titular do Departamento de Energia Nuclear, UFPE. E-mail: salcedo@ufpe.br

(4) Professor Adjunto da Escola de Medicina e Veterinária e Zootecnia da Universidade Federal de Tocantins - UFT . Campus de Araguaína, Br 153, km 112, CEP 77804-970 Araguaina (TO). E-mail: clementino@uft.edu.br
} 


\title{
SUMMARY: CARBON AND NITROGEN FRACTIONS AS AFFECTED BY TEXTURE, RELIEF AND LAND USE IN THE VACA BRAVA WATERSHED
}

\begin{abstract}
Productivity in subsistence or low-input agriculture depends on the release of nutrients through mineralization of soil organic matter (SOM). For this reason, SOM quantity and quality are two important variables related to the sustainability of subsistence family farming. The objective of this work was to study the interrelationships among soil use, texture and relief, at basin scale, on the concentration of total soil $C(C t)$ and $N(N t)$, the oxidizable $C$ fraction with $16.5 \mathrm{~mol} \mathrm{~L}^{-1} \mathrm{KMnO}_{4}$ (Cox) and the amino-sugars fraction of $\mathrm{N}$ (Namino). These properties were quantified in 260 single soil samples (0-20 cm layer) from the Vaca Brava basin, Paraiba State, Brazil. The region was sampled using a stratified sampling design with an incomplete factorial arrangement of the strata in areas under subsistence farming. Concentrations of $\mathrm{Ct}, \mathrm{Nt}$ and Namino decreased in the following order: pasture > fodder > crops. In relation to the relief, $\mathrm{Ct}, \mathrm{Nt}$ and their fractions were greater in lowlands and smaller in foot-hill areas. The latter tended to accumulate sand, while lowlands showed greater accumulation of silt. Sediment redistribution was caused mainly by erosion processes of the back-slope regions, in which finer textures predominated. In relation to textural classes, SOM concentrations and their fractions were greater $(p<0.05)$ in clayey sand samples and smaller in sandy loam ones. In all strata, Cox and Namino concentrations tended to parallel the variations in Ct and Nt. For this reason, Cox/Ct and Nt/Namino ratios that could be used as SOM quality index varied very little as a function of use, relief or texture.
\end{abstract}

Index terms: subsistence farming, soil fertility, pasture, labile fraction of $C$ and $N$.

\section{INTRODUÇÃO}

A conversão de ecossistemas naturais para sistemas agrícolas ou pecuários resulta em declínio da matéria orgânica do solo (MOS), que é acompanhado pela mineralização de nutrientes orgânicos (Voroney et al., 1981; Tiessen et al., 1982, 1992). Este declínio pode ser ainda maior em áreas de relevo ondulado, nas quais se acentuam os processos erosivos (Fraga \& Salcedo, 2004). A produtividade em sistemas agrícolas de subsistência ou de baixos insumos depende do fornecimento de nutrientes provenientes da mineralização da MOS. Portanto, a quantidade e a qualidade da matéria orgânica são duas variáveis fundamentais associadas à sustentabilidade da produção agropecuária de subsistência.

Para acompanhar as mudanças em quantidade e qualidade, vários autores têm enfatizado o fracionamento da MOS, visando à identificação de duas ou três frações com distinta resistência à mineralização (Stevenson, 1994; Blair et al., 1995; Franzluebbers et al., 2000; Khan et al., 2001). A fração mais facilmente mineralizável tem influência sobre a fertilidade do solo por representar um reservatório de nutrientes, sobretudo $\mathrm{N}, \mathrm{P}$ e $\mathrm{S}$, que podem ser liberados para as plantas em curto prazo. A identificação e a quantificação de um reservatório lábil da matéria orgânica por métodos químicos seriam úteis para prever o suprimento potencial de nutrientes durante o ciclo de crescimento de uma cultura (Fraga, 2002). Blair et al. (1995) observaram, em solos cultivados da Austrália, que a redução do $\mathrm{C}$ oxidável foi proporcionalmente maior que a redução do $\mathrm{C}$ total, podendo este $\mathrm{C}$ oxidável atuar como indicador das mudanças atribuídas ao cultivo. Em relação ao $\mathrm{N}$, a mineralização do $\mathrm{N}$ orgânico do solo foi utilizada como um indicador da disponibilidade potencial do $\mathrm{N}$ às culturas (Sampaio et al., 1995a; Camargo et al., 1997; Alves et al., 1999). Métodos químicos também têm sido utilizados com o objetivo de determinar uma fração lábil da MOS, como a fração $\mathrm{N}$ aminoaçúcar (Khan et al., 2001). Pesquisas realizadas em solos do norte-central e nordeste dos Estados Unidos determinaram que áreas de cultivo de milho que não responderam à fertilização nitrogenada apresentavam altos teores de $\mathrm{N}$ aminoaçúcar (Mulvaney et al., 2001; Khan et al., 2001).

Vários trabalhos têm avaliado a MOS lábil por métodos biológicos; no entanto, não foram encontrados estudos que tenham avaliado frações lábeis da MOS por métodos químicos em escala de microbacia, envolvendo variáveis de relevo, de textura e de uso do solo, que constituíram o objetivo do presente trabalho.

\section{MATERIAL E MÉTODOS}

O trabalho foi realizado na microbacia de Vaca Brava, localizada na microrregião do Brejo, na 
mesorregião do Agreste, contraposto oriental do Planalto da Borborema, do Estado da Paraíba, Nordeste do Brasil, compreendida entre as coordenadas (UTM) 192.000 e $198.000 \mathrm{~m} \mathrm{E} \mathrm{e}$ 9.225 .300 e $9.231 .000 \mathrm{~m} \mathrm{~N}$. Segundo a classificação de Köppen, o clima é do tipo As' (tropical chuvoso) (Chaves, 1977). A microbacia ocupa uma superfície de 1.500 ha, com altitude média de $637 \mathrm{~m}$ e temperatura média anual de $24{ }^{\circ} \mathrm{C}$, apresentando pequena amplitude de variação (Santos et al., 2002). Os solos que predominam na bacia são uma associação de Argissolo Vermelho-Amarelo eutrófico e Neossolo Litólico eutrófico e, em menor proporção, o Neossolo Regolítico e o Latossolo Amarelo distrófico (Embrapa, 1999).

A microbacia localiza-se quase que inteiramente no município de Areia-PB e apresenta clima tropical chuvoso (pluviosidade anual de $1.200 \mathrm{~mm}$ ) com chuvas de outono - inverno e déficit hídrico de setembro a janeiro, com relevo ondulado a fortemente ondulado. A parte da microbacia que se localiza no município de Remígio (PB) apresenta características edafoclimáticas de transição, com solos arenosos, relevo menos declivoso e marcante declínio na pluviosidade anual $(820 \mathrm{~mm})$ (Silva et al., 1993), com déficit hídrico de setembro a fevereiro.

A microbacia apresenta dois tipos de uso da terra predominantes, uma área de reserva ecológica (mata nativa) e outra de agropecuária familiar. $\mathrm{Na}$ área sob influência antrópica, com aproximadamente 794 ha (Santos et al., 2002), encontram-se atualmente cinco tipos de uso do solo: capoeira (área de pousio), sabiá (Mimosa caesalpinifolia) (produção de lenha), pastagem, capineira e roçado (agricultura de subsistência e baixa produtividade). A pastagem é o tipo de uso com maior área de abrangência, razão de ser a principal atividade econômica desta região. Apenas os três últimos sistemas de uso foram abordados nesta pesquisa. A coleta de solo (Quadro 1) baseou-se num desenho estratificado de amostragem, com uma combinação fatorial incompleta de estratos, $5 \times 3$, sendo cinco posições no relevo (topo, ombro, meia-encosta, rampa pedimentada e várzea, Figura 1) e três sistemas de uso do solo (roçado, pastagem e capineira). A combinação foi incompleta porque algumas situações de uso não foram encontradas nas cinco posições do relevo.

Em cada área amostral (Quadro 1), georeferenciada, foram retiradas amostras de solo da camada de 0-20 cm de dez pontos, numa área de $10 \times 10 \mathrm{~m}$, e misturadas. Uma vez que essa área amostral $\left(100 \mathrm{~m}^{2}\right)$ foi, pelo menos, duas ordens de magnitude menor que a área representada pela amostra, esta última foi considerada como amostra simples. Durante a amostragem, observou-se um grau elevado de erosão nas encostas.

Quadro 1. Número de amostras de solo por estrato na microbacia Vaca Brava (PB)

\begin{tabular}{|c|c|c|c|c|c|c|}
\hline \multirow{2}{*}{ Uso do solo } & \multirow{2}{*}{ Várzea } & \multicolumn{3}{|c|}{ Encosta } & \multirow{2}{*}{ Topo } & \multirow{2}{*}{ Total } \\
\hline & & Rampa pedimentada & Meia-encosta & Ombro & & \\
\hline Roçado & 16 & 13 & 36 & 9 & 10 & 84 \\
\hline Pastagem & 19 & 17 & 74 & 10 & 13 & 133 \\
\hline Capineira & 16 & 6 & 19 & - & 2 & 43 \\
\hline Total & 51 & 36 & 129 & 19 & 25 & 260 \\
\hline
\end{tabular}

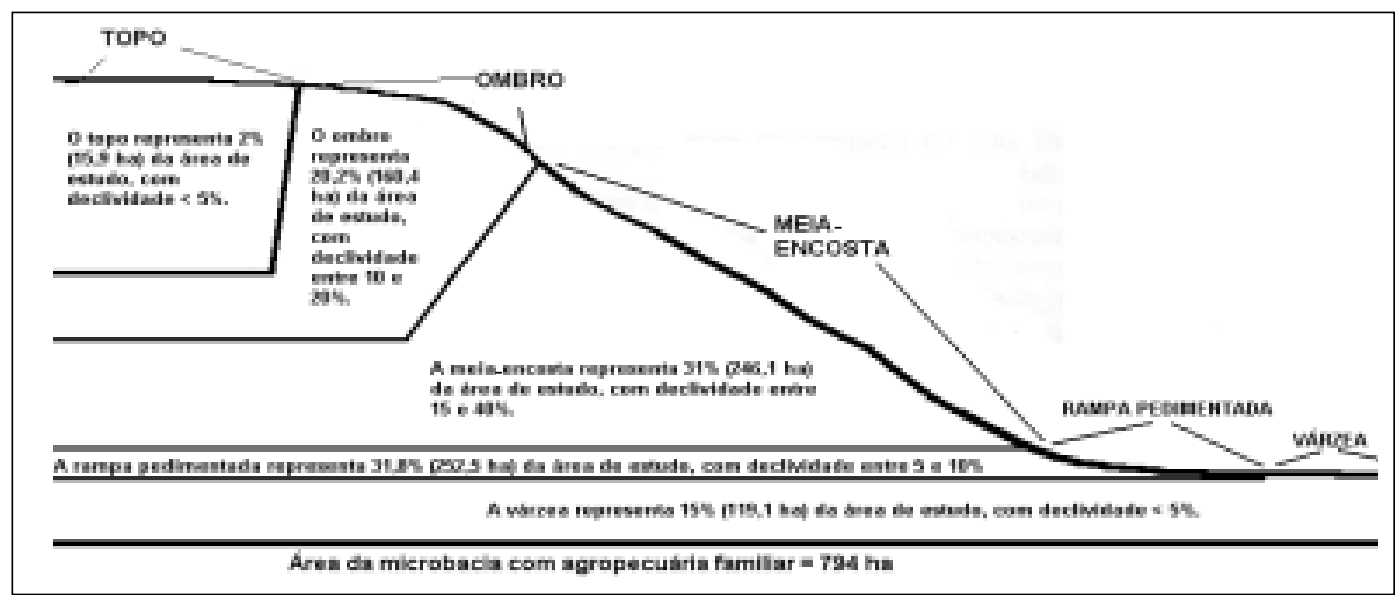

Figura 1. Perfil esquemático das diferentes posições no relevo, seus percentuais e declividades na microbacia Vaca Brava (Santos et al., 2002). 
As amostras foram secas ao ar e passadas por peneira de $2 \mathrm{~mm}$. A granulometria foi determinada pelo método do densímetro, descrita por Day (1965). As análises químicas realizadas foram: C orgânico total, determinado pelo método de oxidação úmidadifusão (Snyder \& Trofymow, 1984); C oxidável de acordo com o método de Blair et al. (1995) modificado por Fraga (2002); N total, pelo método de Kjeldahl por destilação com arraste de vapor (Bremner \& Mulvaney, 1982) e N aminoaçúcar, determinado de acordo com método de Khan et al. (2001).

As variáveis quantitativas apresentaram distribuição log-normal e, por esse motivo, foram apresentadas médias geométricas. O intervalo de confiança (95\%) para essas médias, que é assimétrico, foi calculado, utilizando-se o método proposto por Parkin et al. (1990). Vale salientar que, na maioria dos casos, a média geométrica foi menor que o limite inferior do intervalo. A análise de variância (ANOVA) foi realizada com as variáveis transformadas, utilizando-se um esquema fatorial incompleto $5 \times 3 \times 5$ (cinco posições no relevo, três sistemas de uso e cinco classes texturais) (SAS, 1998). As posições no relevo deram origem a variações texturais marcantes, motivo pela qual esta variável foi incorporada ao desenho experimental na análise dos dados. As médias foram comparadas pelo teste de Tukey a $5 \%$ (SAS, 1998).

\section{RESULTADOS E DISCUSSÃO}

\section{Variações em escala de microbacia}

Os teores de Ct nas amostras oscilaram entre 4,3 e $34,3 \mathrm{~g} \mathrm{~kg}^{-1}$, enquanto a faixa de variação do Cox ficou entre 0,229 e 2,02 $\mathrm{g} \mathrm{kg}^{-1}$. Em média, a relação percentual Cox/Ct foi de $6,09 \%$ e oscilou entre 3,07 e 9,80\% (Quadro 2). Apesar dessa oscilação, $71 \%$ das amostras apresentaram Cox/Ct entre o mínimo e a média. A relação Cox/Ct média deste trabalho foi semelhante à determinada por Fraga (2002), 6,6 \%, em amostras de solo do semiárido. Esta semelhança não era esperada por se tratar de amostras de regiões climáticas relativamente diferentes. Além disto, a granulometria utilizada por Fraga (2002) $(<0,50 \mathrm{~mm})$ foi menor que a utilizada no presente trabalho $(<2 \mathrm{~mm})$, o que, teoricamente, viria expor maior superfície de intra-agregados e aumentaria o teor de matéria orgânica oxidável. Não foi possível comparar a relação Cox/Ct deste trabalho com a de outros autores que utilizaram esse método (Blair et al., 1995; Shang \& Tiessen, 1997), uma vez que utilizaram soluções de permanganato mais concentradas.

Os teores de Nt variaram de 0,39 a 2,61 $\mathrm{g} \mathrm{kg}^{-1}$, enquanto o $\mathrm{N}$ amino variou de 0,020 a $0,20 \mathrm{~g} \mathrm{~kg}^{-1}$ (Quadro 2). Em média, a relação percentual $\mathrm{N}$ amino/Nt foi de $6,7 \%$ do $\mathrm{Nt}$, com uma faixa de variação entre 3,6 e 11 \% (Quadro 2).

A fração de $\mathrm{N}$ amino foi utilizada para identificar solos com distintas intensidades de resposta à fertilização nitrogenada pelo milho (Khan et al., 2001; Mulvaney et al., 2001). Esses autores determinaram que, quando as concentrações de $\mathrm{N}$ amino oscilaram entre 0,303 e $0,511 \mathrm{~g} \mathrm{~kg}^{-1}$, a resposta do milho foi menor do que quando oscilaram entre 0,046 e $0,194 \mathrm{~g} \mathrm{~kg}^{-1}$. Quase todos os valores de $\mathrm{N}$ amino encontrados nas amostras coletadas na microbacia (0,020 e 0,20 $\mathrm{g} \mathrm{kg}^{-1}$, Quadro 2) ficaram na faixa de solos deficientes em $\mathrm{N}$ determinada por Khan et al. (2001) e na sugerida por Mulvaney et al. (2001). Entretanto, deve-se destacar que os valores de $\mathrm{Nt}$ do presente trabalho $\left(0,39-2,61 \mathrm{~g} \mathrm{~kg}^{-1}\right)$ e do trabalho de Khan et al. (2001) (0,61-3,56 $\left.\mathrm{g} \mathrm{kg}^{-1}\right)$ tiveram faixas de variação semelhantes. Por esse motivo, as relações $\mathrm{N}$ amino/Nt dos solos da região temperada que não responderam à fertilização nitrogenada oscilaram entre 12 e $32 \%$ (Khan et al., 2001), enquanto a totalidade das amostras do presente trabalho oscilou entre 3,6 e $11 \%$.

Quadro 2. Médias geométricas, limites de confiança $(p<0,05)$ e valores mínimos e máximos de $\mathrm{C}$ total (Ct), $\mathrm{N}$ total (Nt), C oxidável (Cox), $\mathrm{N}$ aminoaçúcar ( $\mathrm{N}$ amino), Cox/Ct, $\mathrm{N}$ amino/ $\mathrm{Nt}$ e $\mathrm{Ct} / \mathrm{Nt}$ em amostras de solo $(0-20 \mathrm{~cm})$ da microbacia Vaca Brava $(\mathrm{PB})(\mathrm{n}=\mathbf{2 6 0})$

\begin{tabular}{|c|c|c|c|c|}
\hline Variável & Média geométrica & Limites de confiança & Mínimo & Máximo \\
\hline $\mathrm{Ct}\left(\mathrm{g} \mathrm{kg}^{-1}\right)$ & 10,7 & $11,0-12,2$ & 4,30 & 34,3 \\
\hline $\operatorname{Cox}\left(\mathrm{g} \mathrm{kg}^{-1}\right)$ & 0,654 & $0,672-0,745$ & 0,229 & 2,02 \\
\hline $\mathrm{Cox} / \mathrm{Ct}(\%)$ & 6,09 & $6,06-6,39$ & 3,07 & 9,80 \\
\hline $\mathrm{Nt}\left(\mathrm{g} \mathrm{kg}^{-1}\right)$ & 0,89 & $0,91-1,00$ & 0,396 & 2,61 \\
\hline $\mathrm{N}$ amino $\left(\mathrm{g} \mathrm{kg}^{-1}\right)$ & 0,060 & $0,062-0,069$ & 0,020 & 0,201 \\
\hline $\mathrm{N}$ amino/Nt (\%) & 6,72 & $6,71-7,10$ & 3,59 & 10,9 \\
\hline $\mathrm{Ct} / \mathrm{Nt}$ & 12,1 & $12,0-12,3$ & 9,05 & 14,7 \\
\hline Areia (g kg-1) & 712 & $708-738$ & 346 & 885 \\
\hline Silte $\left(\mathrm{g} \mathrm{kg}^{-1}\right)$ & 92,4 & $93-102$ & 38 & 255 \\
\hline Argila (g kg-1) & 156 & $168-194$ & 26 & 569 \\
\hline
\end{tabular}


É possível que as maiores taxas de mineralização da matéria orgânica que normalmente ocorrem em solos da região tropical em relação à região temperada (Moreira \& Siqueira, 2002), associadas à labilidade da fração $\mathrm{N}$ amino, sejam responsáveis pela menor relação $\mathrm{N}$ amino/Nt determinada nas amostras de solos da microbacia. Considerando as diferenças entre solos, clima e vegetação, não é possível assegurar que as faixas de suficiência de $\mathrm{N}$ amino determinadas por Khan et al. (2001) e Mulvaney et al. (2001) sejam aplicáveis aos solos da presente pesquisa.

Com base em experimentos de incubação com solos de região semi-árida e subúmida (Alves et al., 1999) e de ensaio em vasos com 20 solos da região semi-árida (Sampaio et al., 1995b), foi estimado que em torno de $4,8 \%$ do $\mathrm{Nt}$ do solo pode ser mineralizado em um período de quatro a cinco meses (Fraga, 2002). Uma vez que esse percentual é da mesma ordem de magnitude que o valor médio de $6,7 \%$ de $\mathrm{N}$ amino/Nt do presente trabalho, é possível pensar que esta fração possa ser útil para o diagnóstico da fertilidade de solos da região, admitindo-se que a fração tenha a mesma disponibilidade para as plantas determinada por Khan et al (2001).

\section{Efeitos do uso do solo, relevo e classes texturais}

Como não houve interação entre uso do solo, posição no relevo e textura nos teores de $\mathrm{Ct}, \mathrm{Nt}, \mathrm{Cox}$, $\mathrm{N}$ amino, $\mathrm{Ct} / \mathrm{Nt}$, Cox/Ct e $\mathrm{N}$ amino/Nt, os resultados foram discutidos com base no efeito principal de cada variável qualitativa.

Os teores de Ct, $\mathrm{Nt}, \mathrm{N}$ amino ficaram na ordem pastagem $>$ roçado $(p<0,05)$, enquanto a capineira ocupou posição intermediária (Quadro 3). O teor de Cox não diferiu entre sistemas agrícolas, demonstrando menor sensibilidade às mudanças de uso do solo do que a fração $\mathrm{N}$ amino. Diminuição nos teores de $\mathrm{Nt}$ com o aumento da intensidade de uso do solo também foi observada nas mesorregiões do Curimataú e Agreste da Paraíba, onde áreas de pasto e palma apresentaram 0,7 e $0,5 \mathrm{~g} \mathrm{~kg}^{-1} \mathrm{de} \mathrm{N}$ no solo nas de roçado, (Menezes et al., 2002). Os menores valores de $\mathrm{Ct}, \mathrm{Nt}, \mathrm{N}$ amino, encontrados no roçado, deveram-se, provavelmente, aos baixos aportes de resíduos orgânicos, normalmente utilizados na alimentação do gado, bem como ao revolvimento periódico do solo associado ao cultivo de subsistência, que aumenta as taxas médias de mineralização da matéria orgânica (Fraga \& Salcedo, 2004). As gramíneas perenes presentes nas pastagens e capineiras apresentam sistema radicular abundante e elevada rizodeposição, o que favorece a manutenção dos teores de matéria orgânica (Islam \& Weil, 2000).

A razão percentual $\mathrm{N}$ amino/ $\mathrm{Nt}$ foi maior $(\mathrm{p}<0,05)$ na capineira $(6,96 \%)$ e na pastagem $(7,00 \%)$ do que no roçado $(6,20 \%)$, enquanto a razão percentual Cox/Ct não diferiu entre usos do solo (Quadro 3). Por tratar-se de quociente entre uma fração lábil e o total, essas duas razões foram consideradas como índices da qualidade da matéria orgânica do solo. A mudança na qualidade foi observada unicamente para o $\mathrm{N}$ e, embora significativa, foi muito reduzida.

De forma geral, o relevo afetou os teores das variáveis estudadas $(p<0,05)$ (Quadro 4$)$. Os maiores teores médios de $\mathrm{Ct}$ e Nt foram encontrados nas posições de topo, meia-encosta e várzea, enquanto os menores valores foram observados na posição de rampa pedimentada (Quadro 4). A fração $\mathrm{N}$ amino acompanhou as variações de $\mathrm{Nt}$ com o relevo, motivo pelo qual a relação $\mathrm{N}$ amino/Nt não diferiu com o relevo. A fração Cox apresentou o maior valor médio nas áreas de várzea e o menor nas de rampa pedimentada, coincidindo com Ct. Nas posições restantes, a fração Cox não acompanhou os valores de $\mathrm{Ct}$, produzindo-se variações significativas da relação Cox/Ct com o relevo (Quadro 4).

As variações nos teores de MOS e de suas frações, resultantes das mudanças texturais, foram resumidas no quadro 5. A textura argilosa não foi incluída na ANOVA, em razão do pequeno número de amostras com esta textura. O maior teor de $\mathrm{Ct}$ $(\mathrm{p}<0,05)$ foi observado nas amostras de textura argilo-arenosa (18,5 $\left.\mathrm{g} \mathrm{kg}^{-1}\right)$, e o menor nas de textura

Quadro 3. Médias geométricas de $\mathbf{C}$ total $(\mathrm{Ct}), \mathbf{C}$ oxidável $(\mathrm{Cox}), \mathbf{N}$ total $(\mathrm{Nt}), \mathbf{N}$ aminoaçúcar $(\mathbf{N}$ amino), $\mathrm{Cox} / \mathrm{Ct}, \mathrm{N}$ amino/ $\mathrm{Nt}$ e $\mathrm{Ct} / \mathrm{Nt}$ em amostras de solo $(0-20 \mathrm{~cm})$, considerando os diferentes sistemas de uso do solo da microbacia Vaca Brava (PB)

\begin{tabular}{|c|c|c|c|c|c|c|c|}
\hline Uso do solo & $\mathbf{C t}$ & Cox & $\mathbf{N t}$ & $\mathbf{N}$ amino & $\operatorname{Cox} / \mathbf{C t}$ & $\mathbf{N} \operatorname{amino} / \mathbf{N t}$ & $\mathbf{C t} / \mathbf{N t}$ \\
\hline & \multicolumn{4}{|c|}{$\mathrm{g} \mathrm{kg}^{-1}$} & \multicolumn{2}{|c|}{$\%$} & \\
\hline Capineira $(n=43)$ & $10,5 \mathrm{ab}$ & $0,68 \mathrm{a}$ & $0,88 \mathrm{ab}$ & $0,061 \mathrm{a}$ & $6,46 \mathrm{a}$ & $6,96 \mathrm{a}$ & $12,0 \mathrm{a}$ \\
\hline Pastagem $(\mathrm{n}=133)$ & $11,4 \mathrm{a}$ & $0,67 \mathrm{a}$ & $0,94 \mathrm{a}$ & $0,065 \mathrm{a}$ & $5,90 \mathrm{a}$ & $7,00 \mathrm{a}$ & $12,1 \mathrm{a}$ \\
\hline Roçado $(\mathrm{n}=84)$ & $9,92 \mathrm{~b}$ & $0,62 \mathrm{a}$ & $0,82 \mathrm{~b}$ & $0,051 \mathrm{~b}$ & $6,20 \mathrm{a}$ & $6,20 \mathrm{~b}$ & $12,2 \mathrm{a}$ \\
\hline
\end{tabular}

Letras iguais, na coluna, não diferem entre si a $5 \%$ pelo teste de Tukey. 
areia franca $\left(7,45 \mathrm{~g} \mathrm{~kg}^{-1}\right)$. A variável Nt e as frações Cox e $\mathrm{N}$ amino comportaram-se de forma semelhante, apresentando maiores teores nas amostras de textura argilo-arenosa e franco-argiloarenosa e menores teores nas amostras de textura areia franca.

Em relação aos índices de qualidade, apenas a razão Cox/Ct variou significativamente com a textura, sendo maior nas amostras de textura areia franca, intermediária nas de textura franco-arenosa e menor nas de textura argilo-arenosa e francoargilo-arenosa, indicando que menor proporção do $\mathrm{Ct}$ foi oxidada à medida que os teores de argila aumentavam (Quadro 5). Essa crescente resistência à oxidação pode originar-se da proteção que a argila proporciona à MOS, pela elevada superfície específica envolvida e pela forte interação entre os colóides orgânicos e inorgânicos (Bayer, 1996; Shang \& Tiessen, 1997). A proporção $\mathrm{N}$ amino/Nt não foi afetada $(\mathrm{p}<0,05)$ pelas variações texturais (Quadro 5), o que significa que o $\mathrm{Nt}$ e $\mathrm{N}$ amino variaram proporcionalmente com a textura.

Como houve inter-relações significativas entre o relevo e a granulometria (Quadro 6), não foi possível identificar qual desses dois fatores influenciou mais as variações nos teores de matéria orgânica e suas frações (Quadros 4 e 5). Os maiores teores de areia foram encontrados nas áreas de rampa pedimentada e os menores teores nas da meia-encosta (Quadro 6), como resultado dos processos erosivos observados nessas áreas (Santos et al., 2002). Esses processos, atuando em áreas de encosta com solo do tipo Argissolo Vermelho-Amarelo eutrófico que apresenta camada superficial de textura arenosa sobre um horizonte argiloso com transição abrupta (Embrapa, 1999), fez com que as amostras das encostas erodidas apresentassem os maior teores de argila (Quadro 6). A posição de rampa pedimentada, por ser uma área de deposição de partículas mais grosseiras (Gregorich et al., 1998), apresentou os maiores teores de areia, enquanto as partículas menores foram transportadas por distâncias mais longas. Por este motivo o silte tendeu a depositarse na várzea (Quadro 6), enquanto parte da argila foi removida pela rede de drenagem.

Apesar de terem apresentado o menor teor de argila de todas as posições (Quadro 6), as áreas de várzea mostraram os maiores teores de MOS e suas frações (Quadro 4). Por outro lado, embora os teores de argila nas amostras de topo, ombro e meiaencosta tenham sido superiores aos das de várzea (Quadro 6), os teores de Ct, nessas posições, somente

Quadro 4. Médias geométricas de $\mathrm{C}$ total (Ct), $\mathrm{C}$ oxidável (Cox), $\mathbf{N}$ total $(\mathrm{Nt}), \mathbf{N}$ aminoaçúcar ( $\mathrm{N}$ amino), $\mathrm{Cox} / \mathrm{Ct}, \mathrm{N}$ amino/ $\mathbf{N t}$ e $\mathrm{Ct} / \mathrm{Nt}$ em amostras de solo $(0-20 \mathrm{~cm})$ para as diferentes posições no relevo da microbacia Vaca Brava (PB)

\begin{tabular}{|c|c|c|c|c|c|c|c|}
\hline Posição no relevo & $\mathbf{C t}$ & Cox & $\mathbf{N t}$ & $\mathbf{N}$ amino & $\operatorname{Cox} / \mathbf{C t}$ & $\mathbf{N} \operatorname{amino} / \mathbf{N t}$ & $\mathbf{C t} / \mathbf{N t}$ \\
\hline & \multicolumn{4}{|c|}{$\mathrm{g} \mathrm{kg}^{-1}$} & \multicolumn{2}{|c|}{$\%$} & \\
\hline Topo $(\mathrm{n}=25)$ & $10,9 \mathrm{a}$ & $0,66 \mathrm{ab}$ & $0,87 \mathrm{a}$ & $0,058 \mathrm{ab}$ & $6,04 \mathrm{ab}$ & $6,69 \mathrm{a}$ & $12,5 \mathrm{a}$ \\
\hline Ombro $(\mathrm{n}=19)$ & $10,2 \mathrm{ab}$ & $0,59 \mathrm{ab}$ & $0,85 a b$ & $0,053 \mathrm{ab}$ & $5,83 \mathrm{ab}$ & $6,24 \mathrm{a}$ & $12,0 \mathrm{a}$ \\
\hline Meia Encosta $(n=129)$ & $11,1 \mathrm{a}$ & $0,65 \mathrm{ab}$ & 0,93 a & $0,062 \mathrm{a}$ & $5,87 \mathrm{~b}$ & 6,68 a & $12,0 \mathrm{a}$ \\
\hline Rampa pedimentada $(\mathrm{n}=36)$ & $8,62 \mathrm{~b}$ & $0,54 \mathrm{~b}$ & $0,71 \mathrm{~b}$ & $0,049 \mathrm{~b}$ & $6,28 \mathrm{ab}$ & $6,91 \mathrm{a}$ & $12,1 \mathrm{a}$ \\
\hline Várzea $(\mathrm{n}=51)$ & $11,5 \mathrm{a}$ & $0,77 \mathrm{a}$ & $0,95 \mathrm{a}$ & $0,065 \mathrm{a}$ & $6,67 \mathrm{a}$ & $6,91 \mathrm{a}$ & $12,1 \mathrm{a}$ \\
\hline
\end{tabular}

Letras iguais, na coluna, não diferem entre si a $5 \%$ pelo teste de Tukey.

Quadro 5. Médias geométricas de $\mathbf{C}$ total $(\mathrm{Ct}), \mathrm{C}$ oxidável (Cox, $\mathbf{N}$ total $(\mathrm{Nt}), \mathbf{N}$ aminoáçucar $(\mathrm{N}$ amino), $\mathrm{Cox} / \mathrm{Ct}, \mathrm{N}$ amino/Nt e $\mathrm{Ct} / \mathrm{Nt}$ em amostras de solo para as diferentes classes texturais da microbacia Vaca Brava (PB)

\begin{tabular}{|c|c|c|c|c|c|c|c|}
\hline Textura & $\mathbf{C t}$ & Cox & $\mathbf{N t}$ & $\mathbf{N}$ amino & $\operatorname{Cox} / \mathbf{C t}$ & $\mathbf{N} \operatorname{amino} / \mathbf{N t}$ & $\mathbf{C t} / \mathbf{N t}$ \\
\hline & & 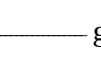 & & - & 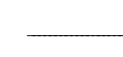 & - & \\
\hline $\operatorname{Arg}(n=06)$ & 18,9 & 0,99 & 1,42 & 0,092 & 5,21 & 6,50 & 13,3 \\
\hline $\operatorname{ArgAr}(n=18)$ & $18,5 \mathrm{a}$ & $0,93 \mathrm{a}$ & $1,44 \mathrm{a}$ & $0,090 \mathrm{a}$ & $5,04 \mathrm{c}$ & $6,24 \mathrm{a}$ & $12,8 \mathrm{a}$ \\
\hline $\operatorname{FrArgAr}(\mathrm{n}=59)$ & $14,2 \mathrm{~b}$ & $0,79 \mathrm{a}$ & $1,17 \mathrm{a}$ & $0,076 \mathrm{a}$ & $5,58 \mathrm{c}$ & $6,56 \mathrm{a}$ & $12,1 \mathrm{ab}$ \\
\hline $\operatorname{FrAr}(\mathrm{n}=99)$ & $10,6 \mathrm{c}$ & $0,66 \mathrm{~b}$ & $0,87 \mathrm{~b}$ & $0,061 \mathrm{~b}$ & $6,19 \mathrm{~b}$ & $6,99 \mathrm{a}$ & $12,2 \mathrm{a}$ \\
\hline $\operatorname{ArFr}(\mathrm{n}=78)$ & $7,45 \mathrm{~d}$ & $0,50 \mathrm{c}$ & $0,64 \mathrm{c}$ & $0,042 \mathrm{c}$ & $6,72 \mathrm{a}$ & $6,65 \mathrm{a}$ & $11,7 \mathrm{~b}$ \\
\hline
\end{tabular}

Letras iguais, na coluna, não diferem entre si a $5 \%$ pelo teste de Tukey.

Arg: argilosa; ArgAr: argilo-arenosa; FrArgAr: franco-argilo-arenosa; FrAr: franco-arenosa; ArFr: areia franca. 
conseguiram alcançar teor semelhante ao de várzea (Quadro 4). As inter-relações entre os teores de Ct, argila e as posições do relevo foram resumidas na figura 2. Os pontos correspondentes às posições de ombro, rampa pedimentada e topo foram ajustados por uma única função de regressão, porque os coeficientes de regressão linear da relação Ct versus argila foram semelhantes $(\mathrm{p}<0,05)$ (Figura 2). As posições restantes apresentaram diferenças significativas entre esses coeficientes. Observa-se, nessa figura, que as amostras de várzea apresentaram maior teor de $\mathrm{Ct}$ que as demais posições para um mesmo teor de argila e um maior acúmulo de Ct por unidade de argila. A relação entre Ct e silte foi significativa apenas na posição da várzea $\left(\mathrm{R}^{2}=0,46\right)$ e o teor de Ct para um mesmo teor de silte foi menor que as das demais posições. De forma geral, os resultados confirmam tendências observadas por outros autores (Woods \& Schuman,

Quadro 6. Médias geométricas dos teores de areia, silte e argila em amostras de solo $(0-20 \mathrm{~cm})$, considerando as diferentes posições no relevo da microbacia Vaca Brava (PB)

\begin{tabular}{llcl}
\hline Posição no relevo & Areia & Silte & Argila \\
& & \multicolumn{3}{c}{$\mathrm{g} \mathrm{kg}^{-1}$} \\
\cline { 2 - 4 } & $718 \mathrm{~b}$ & $68 \mathrm{c}$ & $175 \mathrm{a}$ \\
Topo & $705 \mathrm{bc}$ & $88 \mathrm{~b}$ & $167 \mathrm{a}$ \\
Ombro & $682 \mathrm{c}$ & $90 \mathrm{~b}$ & $187 \mathrm{a}$ \\
Meia-encosta & $790 \mathrm{a}$ & $80 \mathrm{bc}$ & $118 \mathrm{~b}$ \\
Rampa pedimentada & $735 \mathrm{bc}$ & $128 \mathrm{a}$ & $110 \mathrm{~b}$ \\
Várzea & & & \\
\hline
\end{tabular}

Letras iguais, na coluna, não diferem entre si a $5 \%$ pelo teste de Tukey.

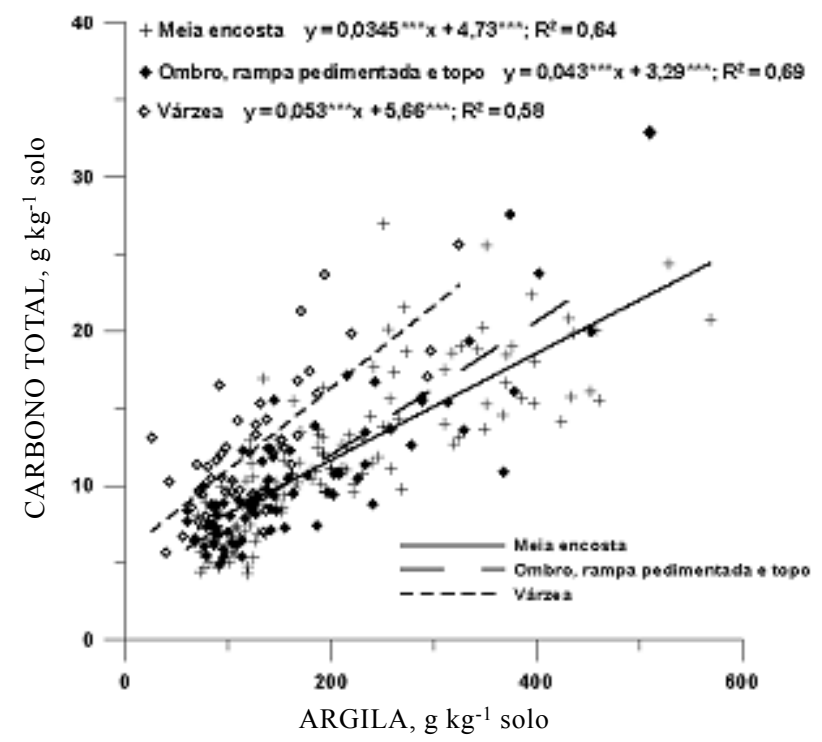

Figura 2. Relação entre $\mathrm{C}$ total e argila de acordo com as posições no relevo.
1998; Gregorich et al., 1998), que apontam as várzeas como área de deposição e os topos e meia-encosta como áreas de erosão.

\section{CONCLUSÕES}

1. O estudo em escala de microbacia permitiu observar, de forma integrada, os principais processos que controlaram a qualidade e quantidade de matéria orgânica na paisagem. Os processos erosivos determinaram redistribuição de sedimentos, que dependendo do tamanho, ocuparam novas posições, ou até, foram perdidos do sistema. A matéria orgânica acompanhou essa redistribuição, sobretudo aquela associada à fração argila. Essa relação entre relevo e textura dificultou atribuir as mudanças em MOS a uma ou outra variável. O uso do solo também foi um fator determinante nas tendências observadas, o roçado tendo propiciado maiores quedas nos teores da MOS, em comparação com o sistema pecuário (pastagens e capineiras).

2. As variações em qualidade, representadas pelos índices $\mathrm{Cox} / \mathrm{Ct}$ e $\mathrm{N}$ amino/Nt, foram evidenciadas por Cox/Ct, para textura e relevo, e por $\mathrm{N}$ amino/Nt, para uso. Entretanto, os intervalos de variação foram sempre muito reduzidos e dificilmente poderiam ter utilidade prática para o diagnóstico de mudanças na qualidade da MOS.

\section{AGRADECIMENTOS}

Os autores agradecem ao Inter American Institute for Global Change (IAI) e ao Conselho Nacional de Desenvolvimento Científico e Tecnológico $(\mathrm{CNPq})$ - Projeto Agricultura Familiar, os recursos recebidos para a execução deste trabalho. Ao Centro de Ciências Agrárias-DSER/UFPB, aos funcionários do Laboratório de RadiagronomiaDEN/UFPE, e aos produtores da Microbacia Vaca Brava, que tornaram esta pesquisa possível.

\section{LITERATURA CITADA}

ALVES, G.D.; SAMPAIO, E.V.S.B.; SALCEDO, I.H. \& SILVA, V.M. Potencial de mineralização de $\mathrm{N}$ e de $\mathrm{C}$ em vinte solos de Pernambuco. R. Bras. Ci. Solo, 23:245-256, 1999.

BAYER, C. Dinâmica da matéria orgânica em sistemas de manejo de solos. Porto Alegre, Universidade Federal do Rio Grande do Sul, 1996. 240p (Tese de Doutorado)

BLAIR, G.J.; LEFROY, D.B. \& LISLE, L. Soil carbon fractions, based on their degree of oxidation, and the development of a carbon management index for agricultural systems. Aust. J. Agric. Res., 46:1459-1466, 1995. 
BREMNER, J.M. \& MULVANEY, C.S. Nitrogen-total. In: PAGE, A.L.; MILLER, R.H. \& KEENEY, D.R., eds. Methods of soil analysis. Chemical and microbiological properties. Madison, Soil Science Society of America, 1982. Part 2. p.595-624.

CAMARGO, F.A.O.; GIANELLO, C. \& VIDOR, C. Potencial de mineralização do nitrogênio orgânico em solos do Rio Grande do Sul. R. Bras. Ci. Solo, 22:575-580, 1997.

CHAVES, I.B. Erosividade das chuvas da microrregião homogênea - n ${ }^{0} 98$ - Estado da Paraíba. Piracicaba, Escola Superior de Agricultura "Luiz de Queiroz", 1977. 78p. (Tese de Mestrado)

DAY, P.R. Particle fracionation and particle size analysis. In: BLAKE, C.A., ed. Method of soil analysis. Madison: American Society of Agronomy, 1965. Part 1. p.545-567.

EMPRESA BRASILEIRA DE PESQUISA AGROPECUÁRIA EMBRAPA. Centro Nacional de Pesquisas de Solos. Sistema Brasileiro de Classificação de Solos. Brasília, 1999. 412p.

FRAGA, V.S. \& SALCEDO, I.H. Declines of organic nutrient pools in tropical semi-arid soils under subsistence farming. Soil Sci. Soc. Am. J., 68:215-224, 2004.

FRAGA, V.S. Mudanças na matéria orgânica (C, N e P) de solos sob agricultura de subsistência. Recife, Universidade Federal de Pernambuco, 2002. 76p. (Tese de Doutorado)

FRANZLUEBBERS, A.J.; HANEY, R.L.; HONEYCUTT, C.W.; SCHOMBERG, H.H.\& HONS, F.M. Flush of carbon dioxide following rewetting of dried soil relates to active organic pools. Soil Sci. Soc. Am. J., 64:613-623, 2000.

GREGORICH, E.G.; GREER, K.J.; ANDERSON, D.W. \& LIANG, B.C. Carbon distribution and losses: Erosion and deposition effects. Soil Till. Res., 47:291-302, 1998.

ISLAM, K.R. \& WEIL, R.R. Land use effects on soil quality in a tropical forest ecosystem of Bangladesh. Agric. Ecosys. Environ., 79:9-16, 2000.

KHAN, S.A.; MULVANEY, R.L. \& HOEFT, R.G. A simple soil test for detecting sites that is nonresponsive to nitrogen fertilization. Soil Sci. Soc. Am. J., 65:1751-1760, 2001.

MENEZES, R.S.C.; TIESSEN, H.; SILVEIRA, L.M.; ANDRADE, L.; SAMPAIO, E.V.S.B.; SALCEDO, I.H.; SABOURIN E.; PORTO, I. \& SARMENTO, C. Balanços de nutrientes em unidades de produção agrícola familiar no Agreste Paraibano: Avaliação e resultados preliminares. In: SILVEIRA, L.; PETERSEN, P. \& SABOURIN, E., eds. Agricultura familiar e agroecologia no semi-árido: avanços a partir do agreste da Paraíba. Rio de Janeiro, AS-PTA, 2002. p.235-248.

MOREIRA, F.M.S. \& SIQUEIRA, J.O. Microbiologia e bioquímica do solo. Lavras, Universidade Federal de Lavras, 2002. 625p.

MULVANEY, R.L.; KHAN, S.A.; HOEFT, R.G. \& BROWN, H.M. A soil organic nitrogen fraction that reduces the need for nitrogen fertilization. Soil Sci. Soc. Am. J., 65:1164-1172, 2001.
PARKIN, T.B.; CHESTER, S.T. \& ROBINSON, J.A. Calculing confidence intervals for the mean of a lognormally distributed variable. Soil Sci. Soc. Am. J., 54:321-326, 1990.

SAMPAIO, E.V.S.B.; SALCEDO, I.H. \& SILVA, F.B.R. Fertilidade de solos do semi-árido do nordeste. In: REUNIÃO BRASILEIRA DE FERTILIDADE DO SOLO E NUTRIÇÃO DE PLANTAS, Petrolina, 1995. Fertilizantes: insumo básico para agricultura e combate à fome. Petrolina, Embrapa, 1995a. p.51-72.

SAMPAIO, E.V.S.B.; SALCEDO, I.H.; SILVA, V.M. \& ALVES, G.D. Capacidade de suprimento de nitrogênio e resposta à fertilização de vinte solos de Pernambuco. R. Bras. Ci. Solo, 19:269-279, 1995b.

SANTOS, A.C.; SALCEDO, I.H. \& CANDEIAS, A.L.B. Relação entre o relevo e as classes texturais do solo na microbacia de Vaca Brava (PB). R. Bras. Cartog., 54:86-94, 2002.

SAS INSTITUTE. Statistical analysis systems (software). Cary, 1998.

SILVA, F.B.R.; RICHÉ, G.R.; TONNEAU, J.P.; SOUSA NETO, N.C.; BRITO, L.T.L.; CORREIA, R.C.; CAVALCANTI, A.C.; SILVA, F.H.B.B.; SILVA, A.B. \& ARAÚJO FILHO, J.C. Zoneamento agroecológico do Nordeste: Diagnóstico do quadro natural e agrossocioeconômico. Petrolina, EmbrapaCPATSA/Recife, Embrapa-CNPS. Coordenadoria Regional Nordeste, 1993. 2v.

SHANG, C. \& TIESSEN, H. Organic matter lability in a tropical Oxisol: Evidence from shifting cultivation, chemical oxidation, particle size, density and magnetic fractionations. Soil Sci., 162:795-807, 1997.

SNYDER, J.D. \& TROFYMOW, J.A. A rapid accurate wet oxidation diffusion procedure for determining organic and inorganic carbon in plant and soil samples. Comm. Soil Sci. Plant Anal., 15:587-597, 1984.

STEVENSON, F.J. Humus chemistry - genesis, composition, reactions. 2.ed. New York, John Wiley \& Sons, 1994. 496p.

TIESSEN, H.; STEWART, W.B. \& BETTANY, J.R. Cultivation effects on the amounts and concentrations of $\mathrm{C}, \mathrm{N}$ and $\mathrm{P}$ in grassland soils. Agron. J., 74:831-835, 1982.

TIESSEN, H.; SALCEDO, I.H. \& SAMPAIO, E.V.S.B. Nutrient and soil organic matter dynamics under shifting cultivation in semi-arid northeastern Brazil. Agric. Ecosyst. Environ., 38:139-151, 1992.

VORONEY, R.P.; van VEEN, J.A. \& PAUL, E.A. Organic C dynamics in grassland soils. 2. Model validation and simulation of the long-term effects of cultivation and rainfall erosion. Can. J. Soil Sci., 61:211-224, 1981.

WOODS, L.E. \& SCHUMAN, G.E. Cultivation and slope position effects on soil organic matter. Soil Sci. Soc. Am. J., 52:13711376,1988 